Virginia Commonwealth University

VCU Scholars Compass

Chemistry Publications

Dept. of Chemistry

2012

\title{
Wettability of pristine and alkyl-functionalized graphane
}

Davide Vanzo

Virginia Commonwealth University

Dusan Bratko

Virginia Commonwealth University, dbratko@vcu.edu

Alenka Luzar

Virginia Commonwealth University, aluzar@vcu.edu

Follow this and additional works at: http://scholarscompass.vcu.edu/chem_pubs

Cart of the Chemistry Commons

Vanzo, D., Bratko, D., \& Luzar, A. Wettability of pristine and alkyl-functionalized graphane. The Journal of Chemical Physics, 137, 034707 (2012). Copyright (C) 2012 American Institute of Physics.

\section{Downloaded from}

http://scholarscompass.vcu.edu/chem_pubs/57

This Article is brought to you for free and open access by the Dept. of Chemistry at VCU Scholars Compass. It has been accepted for inclusion in Chemistry Publications by an authorized administrator of VCU Scholars Compass. For more information, please contact libcompass@vcu.edu. 


\title{
Wettability of pristine and alkyl-functionalized graphane
}

\author{
Davide Vanzo, Dusan Bratko, ${ }^{\text {a) }}$ and Alenka Luzar ${ }^{\text {b) }}$ \\ Department of Chemistry, Virginia Commonwealth University, Richmond, Virginia 23284-2006, USA
}

(Received 5 May 2012; accepted 16 June 2012; published online 18 July 2012;

publisher error corrected 24 July 2012)

Graphane is a hydrogenated form of graphene with high bandgap and planar structure insensitive to a broad range of chemical substitutions. We describe an atomistic simulation approach to predict wetting properties of this new material. We determine the contact angle to be $73^{\circ}$. The lower hydrophobicity compared to graphene is explained by the increased planar density of carbon atoms while we demonstrate that the presence of partial charges on carbon and hydrogen atoms plays only a minor role. We further examine the effects of graphane functionalization by alkyl groups of increasing chain lengths. The gradual increase in contact angle with chain length offers a precise control of surface wettability. A saturated contact angle of $114^{\circ}$ is reached in butylated form. We find the saturation of contact angle with respect to the length of the functional groups to coincide with the loss of water's ability to penetrate the $n$-alkyl molecular brush and interact with carbon atoms of the underlying lattice. Since no experimental data have yet become available, our modeling results provide the first estimate of the wettability of graphane. The results also show how its alkyl functionalization provides the basis for a variety of chemical modifications to tune hydrophilicity while preserving the planar geometry of the substrate. () 2012 American Institute of Physics. [http://dx.doi.org/10.1063/1.4732520]

\section{INTRODUCTION}

Low-dimensional materials such as graphene, ${ }^{1}$ a flat monolayer of carbon atoms packed into a hexagonal lattice, are attracting a growing scientific interest due to their peculiar electronic ${ }^{1-4}$ and chemical properties. ${ }^{5,6}$ More recently, the discovery of a fully hydrogenated form of graphene termed graphane, ${ }^{7,8}$ opened a new and exciting research field for carbon-based nanostructures. Graphane is obtained by hydrogenation on both sides of a graphene sheet. It has been first predicted by Sofo et al. using density-functional theory (DFT) calculations, ${ }^{9}$ showing the higher stability of the chairlike conformer compared to the metastable boat conformation characterized by stronger hydrogen repulsion. The change of carbon hybridization strongly affects the electronic transport properties. With a calculated bandgap of $5.4 \mathrm{eV}$ and $4.9 \mathrm{eV}$ for the chair and boat conformations, respectively, ${ }^{10}$ graphane behaves as large-band insulator. Its stability at ambient temperature enabled synthesis of low-defects graphane based on graphene exposure to a cold hydrogen plasma. ${ }^{7}$ The ensuing characterization confirmed calculated properties. ${ }^{9}$ Aside from the intrinsic potential in nanoelectronic applications, because of its high hydrogen content and the reversibility of the hydrogenation process, ${ }^{7}$ it immediately attracted attention for applications in advanced hydrogen storage technologies. ${ }^{11,12}$ The two-dimensional hydrocarbon nature of graphane opens the possibility of creating a broad range of extended planar molecules through chemical functionalization. Substitution by halides $(-\mathrm{Cl},-\mathrm{F})$ and hydroxyl groups $(-\mathrm{OH})$ are already known. ${ }^{13}$ This unique property makes graphane suitable for

\footnotetext{
a)Electronic mail: dbratko@vcu.edu.

b) Electronic mail: aluzar@vcu.edu.
}

a broad range of applications, above and beyond those found for graphene itself. ${ }^{12}$

Besides the practical applications, graphane also represents an ideal system for theoretical investigation of functionalized surfaces. In general, graphene has become the de facto standard for the modeling of flat and uniform surfaces in computer simulations studies. ${ }^{14,15}$ However its functionalization implies the formations of wrinkles due to the change in hybridization of the functionalized carbon atom. Due to the complete saturation, graphane planarity is unaffected upon the addition of functionalizing groups on either side of the layer. Insulating properties of graphane make it a suitable prototypical material for simulations in the presence of highly charged particles (i.e., ions), without invoking polarizable force-fields required in order to take into account the polarization of the highly conductive graphene surface.

Many potential applications require the interaction with aqueous media, which can be tailored by means of a proper surface functionalization. As an example, a recent work by Yuk et al. ${ }^{16}$ exploited the tendency of water molecules to enter between graphene layers rather than simply laying on them to produce liquid cells for transmission electron microscopy. In this way the inserted droplet minimizes the water-air interface replacing it with more favorable water-graphene interface. As it is expected to be more hydrophilic, graphane appears an even better candidate to support this phenomenon. For these reasons, the characterization of graphane wetting properties holds a great importance for the design strategies of material functionalization.

To the best of our knowledge, no data have yet been reported concerning the contact angle measurement on graphane surfaces. The synthesis problems have so far precluded the formation of graphane sheets large enough to experimentally evaluate the water contact angle on it. Aside 
from this limitation, the measurement of such a parameter on atomically thick surfaces remains quite challenging, as demonstrated by the experimentally available values for graphene. The measurement on silicon deposited graphene sheets ${ }^{17}$ reported water contact angle of $127^{\circ} \pm 4^{\circ}$. This strong hydrophobicity can be rationalized by the presence of roughness due to the superimposition and layering of the deposited flakes. More recently, Shin et al. ${ }^{18}$ measured a $92^{\circ} \pm 3^{\circ}$ contact angle for flat $\mathrm{SiC}$ epitaxially grown graphene. However, this value does not reflect the contact angle of a free standing graphene sheet either due to the vacancies created by the underlying $\mathrm{SiC}$ substrate. ${ }^{19}$

Computer simulations provide a reliable and flexible tool to explore surface properties of these carbon based nanostructures with complete control over the system in question. The goal of the present work is to get insights in wetting properties of pristine graphane surfaces by means of droplet spreading simulations. We evaluated the wettability by measuring the contact angle of cylindrical water drops, enabling direct comparison with macroscopic contact angles measured on graphene surfaces. ${ }^{17-19}$ We find graphane to be considerably more hydrophilic than graphene, primarily due to its area contraction associated with tetrahedral coordination of $s p 3$ carbon atoms. We also examine the use of alkyl chains at increasing length as functionalizing groups suitable to tune hydrophobicity in a systematic manner.

\section{MODELS AND METHODS}

\section{A. Model}

The surface consists of a single $12.4 \mathrm{~nm} \times 19.1 \mathrm{~nm}$ graphane sheet. The lattice parameters have been obtained from previous $a b$ initio calculations ${ }^{9,20}$ which showed good agreement with the subsequent experimental results. ${ }^{7}$ Figure 1 shows the graphane structure in its chair conformation. The change of carbon hybridization involved for graphene hydrogenation implies a stretch of the $\mathrm{C}-\mathrm{C}$ bond length from $1.420 \AA$ for graphene to $1.526 \AA$ for graphane. Together with a $\mathrm{C}-\mathrm{H}$ bond length of $1.110 \AA$ and bond angles of $102.8^{\circ}$ and $107.05^{\circ}$ for $\mathrm{C}-\mathrm{C}-\mathrm{C}$ and $\mathrm{C}-\mathrm{C}-\mathrm{H}$, respectively, the structural parameters for graphane show a good agreement with the standard values for $s p 3$ hybridization. Despite in-

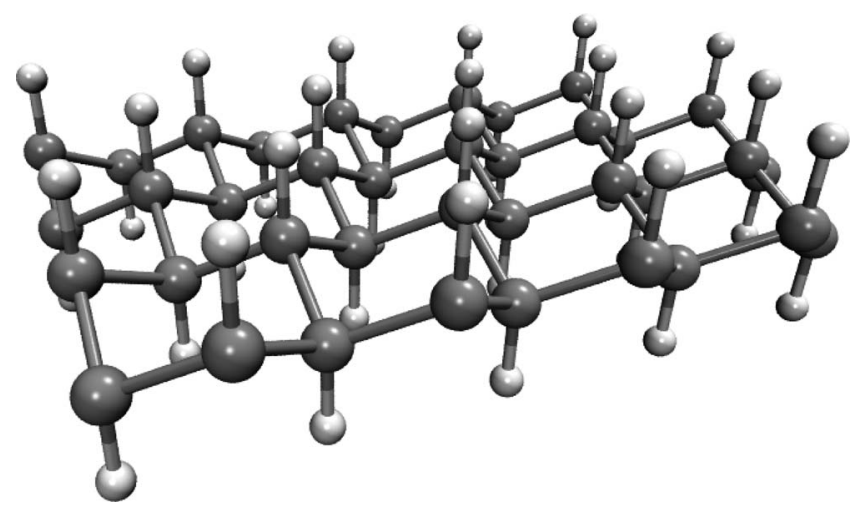

FIG. 1. Atomic structure of graphane in its chair conformation. Carbon and hydrogen atoms are shown in grey and white color, respectively.
TABLE I. OPLS-AA Lennard-Jones parameters and charges ${ }^{25}$ used for $n$-alkyl functionalized graphane simulations.

\begin{tabular}{lccr}
\hline \hline Atom & $\sigma(\AA)$ & $\epsilon\left(\mathrm{kcal} \mathrm{mol}^{-1}\right)$ & $q\left(e_{0}\right)$ \\
\hline $\mathrm{C}, \mathrm{RCH}_{3}$ & 3.500 & 0.066 & -0.180 \\
$\mathrm{C}, \mathrm{R}_{2} \mathrm{CH}_{2}$ & 3.500 & 0.066 & -0.120 \\
$\mathrm{C}, \mathrm{R}_{3} \mathrm{CH}$ & 3.500 & 0.066 & -0.060 \\
$\mathrm{C}, \mathrm{R}{ }_{4} \mathrm{C}$ & 3.500 & 0.066 & 0.000 \\
$\mathrm{H}, \mathrm{RH}$ & 2.500 & 0.030 & 0.060 \\
\hline \hline
\end{tabular}

creased $\mathrm{C}-\mathrm{C}$ bond length, the projected in-plane $\mathrm{C}-\mathrm{C}$ distance of graphane is smaller than in graphene because of tetrahedral carbon coordination, leading to stronger attraction with water.

Surface hydrophobicity is controlled by functionalization with flexible $n$-alkyl groups of general formula $-\mathrm{C}_{n} \mathrm{H}_{(2 n+1)}$ at different chain lengths, from $n=0$ to $n=8$. The number density per surface area is $4.01 \mathrm{~nm}^{-2}$, similar to the typical average surface density of alkanethiols self-assembled monolayers (SAMs). ${ }^{21-24}$ We place alkyl chains on the surface as uniformly as possible although an entirely regular pattern cannot be accommodated for functionalization density we use.

We obtain molecular parametrization from the all-atom optimized potentials for liquid simulation force field (OPLSAA) (Ref. 25) and use (SPC/E) model ${ }^{26}$ for water. The LJ parameters and charges employed in this work are reported in Table I. In many of the available force fields, ${ }^{27}$ partial charges are obtained from $a b$ initio calculations on the specific molecule. On the other hand, the OPLS-AA force field partial charges for a specific moiety are estimated empirically in order to accurately reproduce the conformational and thermodynamic properties of the corresponding organic liquid. ${ }^{25}$ Specifically for hydrocarbons, empirical partial charges somewhat below the calculated ones are used to offset the effect of exaggerated charge localization in interaction-site models. The parametrization obtained for neutral building blocks of known molecules can then be used to extract force fields of new compounds like graphane.

While the choice of interaction parameters is important to capture correct hydrophobicity, ${ }^{28-30}$ major force fields possess adequate parametrization for hydrocarbons. Due to the non-polar nature of the modeled surfaces, with contact angles in the range $90^{\circ} \pm 20^{\circ}$, our comparisons suggest we can expect at most a very weak dependence of the results on the specific force field. ${ }^{31}$ Heteroatomic pairwise interaction parameters follow geometrical mixing rules, as required for OPLS-AA force field. The use of a non-polarizable force field is expected to give a good approximation of the underlying physics due to the insulating properties of the graphane surface.

\section{B. Methods}

Molecular dynamics: All the simulations are performed by using the large-scale atomic molecular massively parallel simulator (LAMMPS) MD code ${ }^{32}$ in NVT ensemble. The temperature is kept constant at $T=300 \mathrm{~K}$ by means of the implemented Nose-Hoover thermostat ${ }^{33}$ with a relaxation time step 


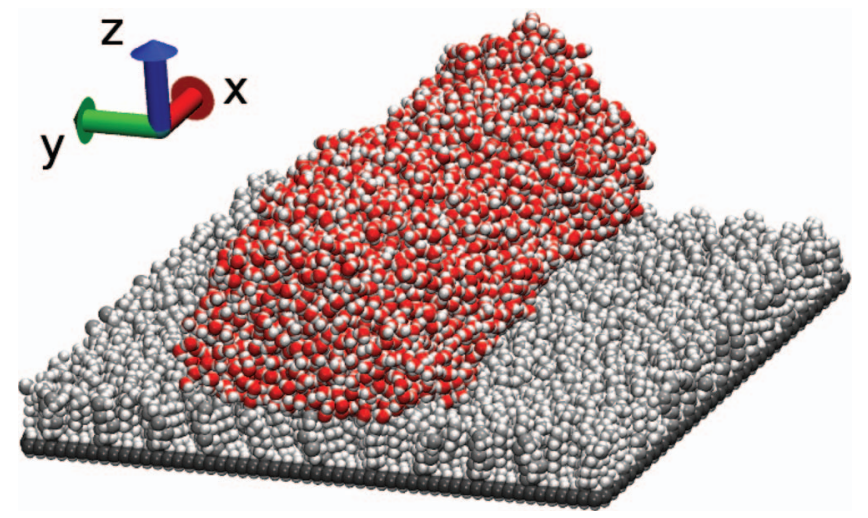

FIG. 2. Visual representation of a fragment of the semi-infinite cylindrical water droplet in contact with the graphene surface fuctionalized with $n$-octyl chains after $1 \mathrm{~ns}$ simulation at $T=300 \mathrm{~K}$.

of 0.1 ps. Verlet integrator is used with a time step size of 1 fs. Non-bonded interactions are calculated by means of a standard 12-6 Lennard-Jones (LJ) potential truncated and shifted at $20 \AA$. The large cutoff is used to eliminate the need for calculating the tail correction for the truncated $\mathrm{LJ}$ potential in a strongly non-uniform system. ${ }^{34}$ Periodic boundary conditions are applied along the three dimensions. The graphane atoms positions are kept fixed during the simulations by zeroing their velocities and forces acting on them. Computational time is also optimized by omitting the calculation of graphane atoms self-interactions. The temperature is therefore calculated only according to the water and alkyl chains atom velocities. Long range electrostatic interactions are computed by means of the particle-particle-particle-mesh solver ${ }^{35}$ with $10^{-5}$ accuracy and a $20 \AA$ real space cutoff.

Contact angle measurement: To simulate the water spreading on graphane surfaces we use a cylindrically shaped sessile nanodroplet ${ }^{36-38}$ with 6578 water molecules, oriented along the $\mathrm{x}$ axis of the reference frame, as shown in Fig. 2. The drop is periodically replicated along the axial direction. Although the classical hemispherical sessile drop is widely employed for the calculation of contact angles in computer simulations, ${ }^{28,31,39-51}$ our current choice has been motivated by two main reasons. At the nanoscale, previous computational investigations for LJ fluid nanodroplets ${ }^{37,52}$ show that, despite a good agreement with the Young equation predictions, small deviations of the contact angles are observed at increasing triple line curvature. Our comparisons between Young contact angles from thermodynamic integration ${ }^{31}$ or pressure tensor calculations ${ }^{53}$ with geometric ones ${ }^{42}$ show deviations of up to $5^{\circ}$. Scocchi et al. ${ }^{38}$ reported that simulated cylindrical nanodroplets of SPC/Fw water molecules ${ }^{54}$ show a negligible dependence of the measured contact angle to both the drop size and the interfacial surface tension. Hemispherical aqueous nanodroplets, on the other hand, show contact angle size dependence for small drops with contact area radius below $r<25 \AA .^{28,42,55}$ Although the size dependence weakens for low interfacial tensions, the cylindrical geometry, characterized by essentially straight contact lines, ensures a higher reliability independently on the system properties.

The second reason for the adoption of a semi-infinite cylindrical drop is the optimization of computation. Since the
LAMMPS simulation engine does not provide a runtime load balancing of the number of atoms assigned to each processor on parallel executions, the simulation of samples with non-uniform density distribution presents very low scalability compared to a bulk system. The use of a cylindrical drop allows us to assign to each processor an even number of atoms by ideally "slicing" the system perpendicularly to the longitudinal axis of the cylindrical drop. Improved computational efficiency permits simulation of bigger cylindrical drops compared to calculations in the hemispherical drop geometry, visibly improving the statistics of contact angle calculation.

To measure the contact angle of our cylindrical sessile drop we employed a specific technique similar to that presented by de Ruijter et ll $^{41}$ for the measurement of dynamic contact angle. Compared to the commonly used technique relying on the fitting of the time averaged density profile of the drop, this method provides a direct insight on the drop spreading dynamics, since the instantaneous contact angle for each configuration is calculated. Each system, consisting of a pre-equilibrated cuboidal water drop situated at about $6 \AA$ above the functionalized surface, is equilibrated with a first energy minimization of the $n$-alkyl chains, followed by a 150 ps $N V T$ run where only the $n$-alkyl chains positions are integrated. The analyzed trajectory is then collected during a $1 \mathrm{~ns}$ simulation run in the canonical ensemble, by storing the system snapshots every $0.5 \mathrm{ps}$. We divided each cylindrical drop in three transversal slices in order to avoid errors in the profile determination due to possible long range oscillations of the triple line. To identify the drop interface for each slice, we use a square binning of local number of water molecules along the $y$ and $z$ directions with a bin size of $2 \AA$. The points defining the equimolar dividing surface are identified as the points where the local density decreases below $50 \%$ from the bulk water density. We determine the contact angle by calculating the best circular fit of the drop density profile according to the method described in Ref. 56. Since a distortion from the circular cross section is known to occur near the surface-water interface, ${ }^{37,41}$ we fit only the contour above the distance that secures convergence of contact angle. We adopted the position identified by half the distance between the two water peaks from the farthest peak with respect to the surface as empirical threshold. Here we refer to the density distribution calculated in a $10 \AA$ thick section of the drop, parallel to the $x$ direction and centered with respect to the drop center of mass. According to the same distribution, we identify the position of the reference contact plane for the contact angle calculation at one radius of oxygen atom below the first oxygen density peak. Although different heights of the contact plane have been used, ${ }^{31,37,57}$ we believe that our choice best represents the average contact height with the mobile $n$-alkyl chains felt by the drop. This assumption is validated by good agreement with the experimental contact angle values measured on $n$-alkanethyols SAMs as we report in the next chapter.

Figure 3 illustrates the temporal contact angle evolution during water drop spreading on pristine $(n=0)$ or functionalized ( $n=2$ and $n=4$ ) graphane surfaces. As expected, contact angle increases with increasing $n$ alkyl chain length. The contact angle converges after about 


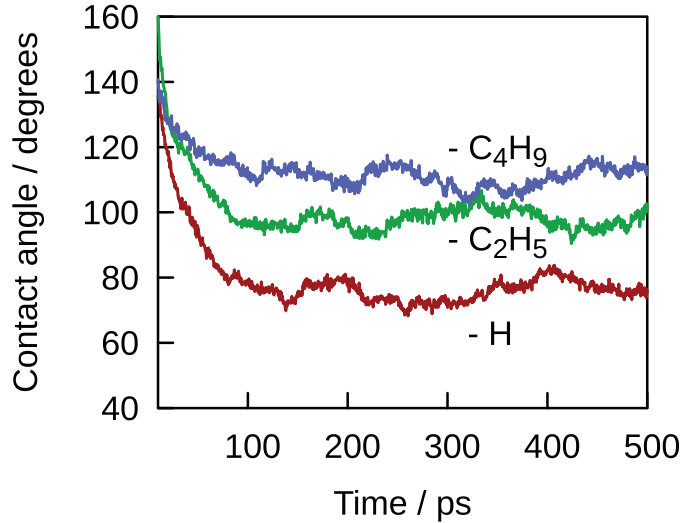

FIG. 3. Contact angle relaxation of a 7000 water molecules cylindrical drop at $300 \mathrm{~K}$ in contact with pristine graphane (bottom) and ethyl (middle) or butyl (top) functionalized graphane surfaces.

$150 \mathrm{ps}$ independently of the nature of the functionalization. To accumulate sufficient contact angle statistics, we calculated the equilibrium contact angle by averaging the instantaneous values obtained from $500 \mathrm{ps}$ to $1 \mathrm{~ns}$ of simulation.

\section{RESULTS AND DISCUSSION}

In Table II we report the average equilibrium contact angles measured for a cylindrical sessile water drop on differently functionalized graphane surfaces. We determine the contact angle of pure graphane to be $73^{\circ} \pm 2^{\circ}$. By repeating the simulation with mobile surface hydrogen atoms we also verified that the impact of atom mobility on the measured contact angle is negligible, with a resulting contact angle of $75^{\circ} \pm 2^{\circ}$. As expected, graphane is more hydrophilic than graphene which, notwithstanding the high spread of experimental data, ${ }^{18,19}$ presents a reproducible simulated contact angle close to $90^{\circ}$. To assess the contribution of electrostatic interactions between water and partial charges present on graphane, but not on graphene, in a separate calculation we remove partial charges on the graphane atoms. The resulting equilibrium contact angle of $74^{\circ} \pm 1^{\circ}$ demonstrates insignificant effects of electrostatic graphane/water interactions in analogy with an earlier observation on fluorocarbon. ${ }^{57}$ The LJ interactions, which drive the spreading, however, intensify due to increased (projected) surface atomic density in

TABLE II. Comparison of the water contact angles on pristine and $n$-alkyl functionalized graphane surfaces with flexible and frozen chains.

\begin{tabular}{lcc}
\hline \hline & \multicolumn{2}{c}{ Contact angle } \\
\cline { 2 - 3 } Functional group & Flexible & Rigid \\
\hline$-\mathrm{H}$ & $75^{\circ} \pm 2^{\circ}$ & $73^{\circ} \pm 2^{\circ}$ \\
$-\mathrm{CH}_{3}$ & $83^{\circ} \pm 3^{\circ}$ & $81^{\circ} \pm 3^{\circ}$ \\
$-\mathrm{C}_{2} \mathrm{H}_{5}$ & $98^{\circ} \pm 4^{\circ}$ & $98^{\circ} \pm 2^{\circ}$ \\
$-\mathrm{C}_{3} \mathrm{H}_{7}$ & $110^{\circ} \pm 3^{\circ}$ & $112^{\circ} \pm 4^{\circ}$ \\
$-\mathrm{C}_{4} \mathrm{H}_{9}$ & $114^{\circ} \pm 4^{\circ}$ & $111^{\circ} \pm 3^{\circ}$ \\
$-\mathrm{C}_{5} \mathrm{H}_{11}$ & $114^{\circ} \pm 3^{\circ}$ & $\ldots$ \\
$-\mathrm{C}_{6} \mathrm{H}_{13}$ & $114^{\circ} \pm 5^{\circ}$ & $\ldots$ \\
$-\mathrm{C}_{7} \mathrm{H}_{15}$ & $115^{\circ} \pm 3^{\circ}$ & $\cdots$ \\
$-\mathrm{C}_{8} \mathrm{H}_{17}$ & $113^{\circ} \pm 3^{\circ}$ & $\cdots$ \\
\hline \hline
\end{tabular}

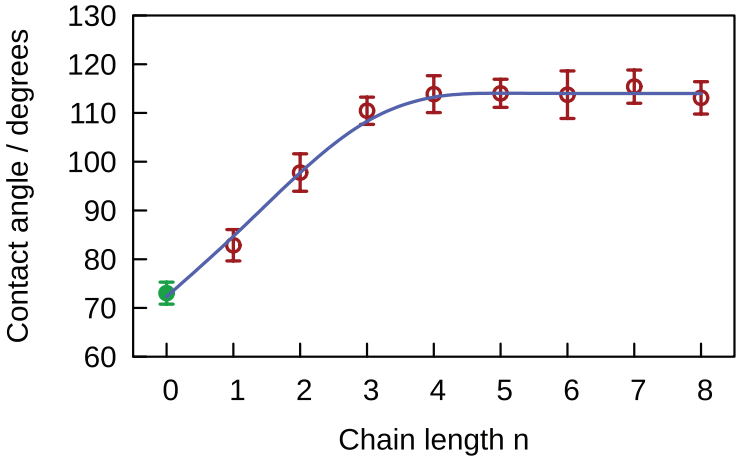

FIG. 4. Equilibrium contact angles of the cylindrical water drop as a function of the degree of functionalization of the graphene surface with $n$-alkyl chains with general formula $-\mathrm{C}_{n} \mathrm{H}_{(2 n+1)}$. The solid green circle represents the pristine graphane surface.

graphane upon hydrogenation of the graphene sheet explaining the increase in hydrophilicity (i.e., decrease in contact angle).

Coverage by methyl groups strongly affects the equilibrium contact angle, which shows a $10^{\circ}$ increase. As evident from Fig. 4, contact angle increases almost linearly with respect to the alkyl chain length until $n=3$, and levels off at about of $114^{\circ}$ thereafter. Our result for long chains is in good agreement with the experimental equilibrium contact angle ${ }^{58,59}$ for water on $-\mathrm{CH}_{3}$ terminating SAMs of about $112^{\circ}$.

We also investigated the effect of chain mobility on the measured equilibrium contact angle. We started a new set of simulations from the final configurations of the previous droplet-spreading simulations, but freezing the positions of the alkyl chains. As reported in Table II, the performed analysis of the contact angle demonstrates that both the average value and the fluctuations of the instantaneous contact angles are insensitive to thermal motion of the chains of the alkyl monolayer.

In order to explain the convergence of the contact angle we calculated water density profiles along the $z$ direction for a $1 \mathrm{~nm}$ thick longitudinal slab of the cylindrical drop, centered on the center of mass of the drop. In Fig. 5 we compare the vertical density profiles of water for the pristine graphane surface and $n=1,2,3,4$ functionalized graphane. The peak structure rapidly decreases due to the increasing distance of the first water layer from the graphane surface. A well defined shoulder at the ascending arm of the first layer peak clearly demonstrates penetration of water molecules in the accessible space between the functionalizing chains.

In view of the non-uniform pattern of the alkyl chains arrangement, the vertical density profiles alone do not provide a complete structural description of the hydration water which also concerns the planar distribution of the penetrating water molecules in the layer. We calculated the surface density distributions of water molecules by binning on a $0.1 \AA$ mesh 2D grid parallel to the $x y$ plane. We monitored the number of water molecules within the layer between the graphane surface and the top of the chains at $\sigma_{H} / 2$ from the highest alkyl hydrogen distribution peak. Due to the low number of water molecules inside the alkyl layer, we collected their 


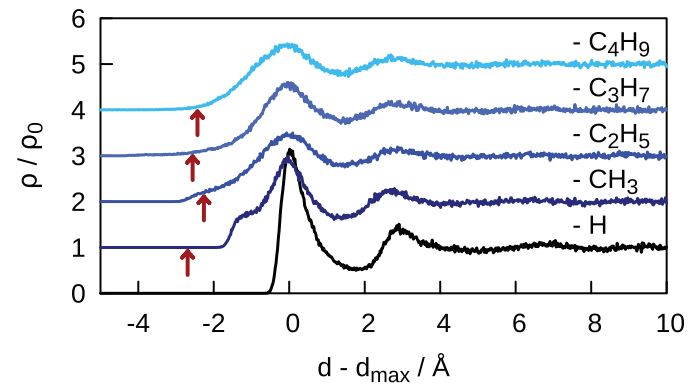

FIG. 5. Vertical density profile of water molecules in the center of cylindrical drops on pristine and functionalized graphane with $n$-alkyl chains at increasing length as function of the distance from the first density peak, at distance $d_{\text {max }}$ from the graphane surface. Red arrows represent the relative first peak position of the $-\mathrm{CH}_{3}$ hydrogen atoms. The distributions are vertically shifted for easier comparison.

positions over 3800 configurations, saved every 0.5 ps. According to the maps reported in Fig. 6 for molecular brushes with $n=2$ and $n=4$, water molecules are distributed almost uniformly between the chains of the functionalizing pattern. However, we observe stronger penetration for the shorter chains, a clear evidence of the contact between the water molecules and the underlying graphane surface. For butyl brush the water density distribution is more uniform due to the loss of contact with graphane, and the higher mobility of the butyl heads. A closer view of the water distribution in Fig. 5 for $-\mathrm{C}_{3} \mathrm{H}_{7}$ functionalization reveals a very smooth shoulder, which is absent from the profiles obtained for longer chains. Convergence of the measured contact angle coincides with the loss of direct contact between water and the underlying graphane surface. At the chain lengths exceeding $n=3$ the free energy penalty due to the hydration of the hydrophobic molecular brush can no more be balanced by the energy gain due to the contact with the high atomic-density graphane surface.

Our results for contact angles at increasing alkyl chain length also demonstrate that the water drop becomes insensitive to the nature of the underlying surface when the chains are longer than $n=3$. At this length, the first peak of calculated density profiles of water molecules, corresponding to the first water layer in contact with the top of the alkyl chains, is located at about $9.2 \AA$ above the middle plane of the graphane surface. This length scale is in good agreement with $\approx 1 \mathrm{~nm}$ crossover from the size-dependent solvation energetics of a small solute and size-insensitive large-solute behavior reported for systems dominated by LJ interaction. ${ }^{60-62}$ This observation represents an additional evidence of the major role of the dispersion forces in determining hydrophobicity/hydrophilicity of our surface. It also suggests that, for the sake of computational optimization, butyl chains can be considered converged with respect to the chain length and hence adequate to simulate SAM layers of lengths sufficient to be insensitive to the specific nature of the supporting surface with short-ranged attraction to water. A bigger saturation length can be expected with highly polarizable substrates such as metals ${ }^{63}$ which support strong dipole-image ${ }^{64}$ interactions.

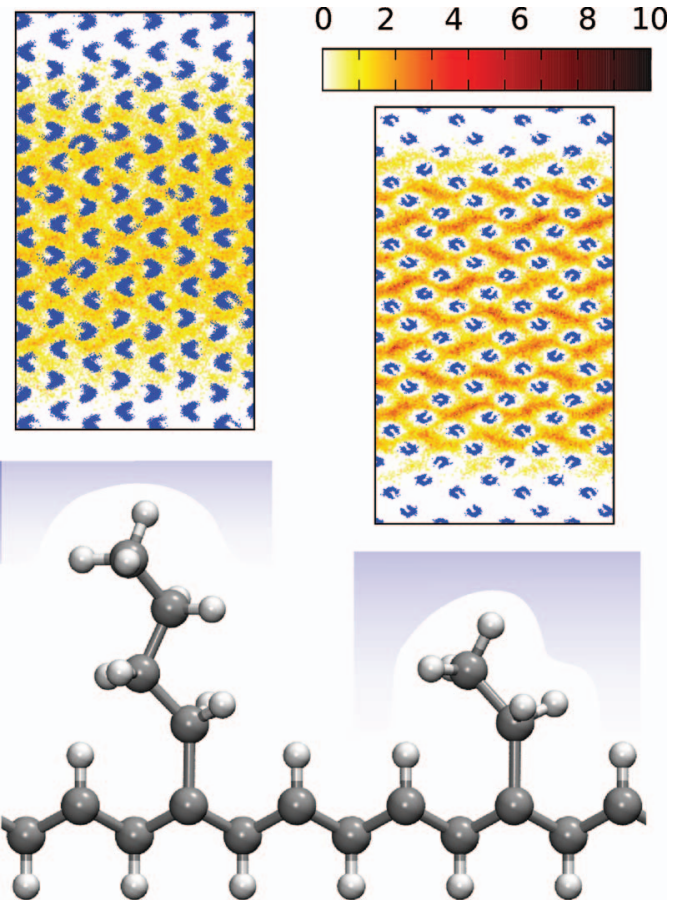

FIG. 6. Illustration of water penetration inside the $-\mathrm{C}_{2} \mathrm{H}_{5}$ and $-\mathrm{C}_{4} \mathrm{H}_{9}$ molecular brushes covalently bonded to a graphane surface. (Top) Surface density maps of water drop molecules inside the volume defined by the graphane surface and the highest average position of the $n$-alkyl chains hydrogens. The resulting thicknesses are $3.35 \AA$ for ethyl chains (right) and $5.87 \AA$ for butyl chains (left). Colorization represents the overall number of molecules in each vertical subvolume with $0.1 \AA x y$ mesh resolution over 3800 system configurations. Blue dots correspond to the distributions of the $-\mathrm{CH}_{3}$ carbon atoms. (Bottom) Visual representation of water penetration on the two molecular brushes.

\section{CONCLUSIONS}

Our modeling results provide the first insights into the wetting ability of graphane, the recently synthesized, fully hydrogenated derivative of graphene. Eliminating the curvature of the three-phase contact line, our cylindrical nanodrop technique provides contact angles essentially free of line tension effects. With contact angle of about $73^{\circ}$, graphane is found to be considerably more hydrophilic than graphene. The difference is associated with increased projected density of carbon atoms as the underlying lattice transforms from planar to tetrahedral coordination along with the transition from $s p 2$ to $s p 3$ hybridization. The latter form, characteristic of graphane, supports surface functionalizations without inducing deformations and wrinkling observed on chemically modified graphene surfaces. Functionalization by alkyl chains brings contact angle closer to that of hydrocarbon surfaces. The gradual increase in contact angle with chain length enables precise tuning of surface wettability until a saturated contact angle is reached in butylated form. At this chain length, the alkyl brush is sufficiently thick to preclude any significant penetration of water and its interaction with the underlying graphane lattice. The wetting properties are therefore converged at significantly shorter tail lengths than in the case of physisorbed SAMs layers where stable coverage is only possible with longer chains. Unlike thick, physisorbed SAMs deposits, the brush of covalently bonded butyl functionalities 
behaves as an essentially incompressible layer whose conformation is not expected to change upon end-group substitutions. As such it provides the basis for a variety of chemical modifications to control the substrate hydrophilicity ${ }^{65}$ while preserving the thickness and planarity of the surface.

\section{ACKNOWLEDGMENTS}

We thank Sebastien Lebegue for sharing his DFT results for graphane partial charges. We gratefully acknowledge the support of the Office of Basic Energy Sciences, Chemical Science, Geosciences, and Biosciences Division of the U. S. Department of Energy (DE-SC-0004406). This research used resources of the National Energy Research scientific Computing Center (NERSC), which is supported by the Office of Science of the U.S. Department of Energy under Contract No. DEAC02-05CH11231, and the Extreme Science and Engineering Discovery Environment (XSEDE), which is supported by National Science Foundation Grant No. OCI-1053575.

${ }^{1}$ A. K. Geim and K. S. Novoselov, Nature Mater. 6, 183 (2007).

${ }^{2}$ K. S. Novoselov, A. K. Geim, S. V. Morozov, D. Jiang, M. I. Katsnelson, I. V. Grigorieva, S. V. Dubonos, and A. A. Frisov, Nature (London) 438, 197 (2005).

${ }^{3}$ Y. Zhang, Y.-W. Tan, H. L. Stromer, and P. Kim, Nature (London) 438, 201 (2005).

${ }^{4}$ D. A. Abanin, P. A. Lee, and L. S. Levitov, Phys. Rev. Lett. 96, 176803 (2006).

${ }^{5}$ F. Schedin, A. K. Geim, S. V. Morozov, E. W. Hill, P. Blake, M. I. Katsnelson, and K. S. Novoselov, Nature Mater. 6, 652 (2007).

${ }^{6}$ P. Hu, J. Zhang, L. Li, Z. Wang, W. O’Neill, and P. Estrela, Sensors 10, 5133 (2010).

${ }^{7}$ D. C. Elias, R. R. Nair, T. M. G. Mohiuddin, S. V. Morozov, P. Blake, M. P. Halsall, A. C. Ferrari, D. W. Boukhvalov, M. I. Katsnelson, A. K. Geim, and K. S. Novoselov, Science 323, 610 (2009).

${ }^{8}$ J. D. Jones, K. K. Mahajan, W. H. Williams, P. A. Ecton, Y. Mo, and J. M. Perez, Carbon 48, 2335 (2010).

${ }^{9}$ J. O. Sofo, A. S. Chaudhari, and G. D. Barber, Phys. Rev. B 75, 153401 (2007).

${ }^{10} \mathrm{~S}$. Lebégue, M. Klintenberg, O. Eriksson, and M. I. Katsnelson, Phys. Rev. B 79, 245117 (2009).

${ }^{11}$ A. Savchenko, Science 323, 589 (2009).

${ }^{12}$ J. Agbenyega, Mater. Today 12, 13 (2009).

${ }^{13}$ J. T. Robinson, J. S. Burgess, C. E. Junkermeier, S. C. Badescu, T. L. Reinecke, F. K. Perkins, M. K. Zalalutdniov, J. W. Baldwin, J. C. Culbertson, P. E. Sheehan, and E. S. Snow, Nano Lett. 10, 3001 (2010).

${ }^{14}$ J. Li, T. Liu, X. Li, L. Ye, H. Chen, H. Fang, Z. Wu, and R. Zhou, J. Phys. Chem. B 109, 13639 (2005).

${ }^{15}$ C. Eun and M. L. Berkowitz, J. Phys. Chem. A 115, 6059 (2011).

${ }^{16}$ J. M. Yuk, J. Park, P. Ericus, K. Kim, D. J. Hellebusch, M. F. Crommie, J. Y. Lee, A. Zettl, and P. A. Alivisatos, Science 336, 61 (2012).

${ }^{17}$ S. Wang, Y. Zhang, N. Abidi, and L. Cabrales, Langmuir 25, 11078 (2009).

${ }^{18}$ Y. J. Shin, Y. Wang, H. Huang, G. Kalon, A. T. S. Wee, Z. Shen, C. S. Bhatia, and H. Yang, Langmuir 26, 3798 (2010).

${ }^{19}$ H. Zhou, P. Ganesh, V. Presser, M. C. F. Wander, P. Fenter, P. R. C. Kent, D.-E. Jiang, A. A. Chialvo, J. McDonough, K. L. Shuford, and Y. Gogotsi, Phys. Rev. B 85, 035406 (2012).

${ }^{20}$ D. W. Boukhvalov, M. I. Katsnelson, and A. I. Lichtenstein, Phys. Rev. B 77, 035427 (2008).

${ }^{21}$ C. D. Bain, B. E. Troughton, Y. T. Tao, J. Evall, G. M. Whitesides, and R. G. Nuzzo, J. Am. Chem. Soc. 111, 321 (1989).

${ }^{22}$ C. D. Bain, J. Evall, and G. M. Whitesides, J. Am. Chem. Soc. 111, 7155 (1989).

${ }^{23}$ C. D. Bain and G. M. Whitesides, J. Am. Chem. Soc. 111, 7164 (1989).
${ }^{24}$ Y. Arima and H. Iwata, Biomaterials 28, 3074 (2007).

${ }^{25}$ W. L. Jorgensen, D. S. Maxwell, and J. Tirado-Rives, J. Am. Chem. Soc. 118, 11225 (1996).

${ }^{26}$ H. J. C. Berendsen, J. R. Grigera, and T. P. Straatsma, J. Phys. Chem. 91, 6269 (1987).

${ }^{27}$ W. D. Cornell, P. Cieplak, C. I. Bayly, I. R. Gould, K. M. Merz, D. M. Ferguson, D. C. Spellmeyer, T. Fox, J. W. Caldwell, and P. A. Kollman, J. Am. Chem. Soc. 117, 5179 (1995).

${ }^{28}$ T. Werder, J. H. Walther, R. L. Jaffe, T. Halicioglu, and P. Koumoutsakos, J. Phys. Chem. B 107, 1345 (2003).

${ }^{29}$ N. Choudhury, J. Phys. Chem. B 112, 6296 (2008).

${ }^{30}$ C. Eun and M. L. Berkowitz, J. Phys. Chem. B 114, 13410 (2010).

${ }^{31}$ J. H. Wang, D. Bratko, and A. Luzar, Proc. Natl. Acad. Sci. U.S.A. 108, 6374 (2011)

${ }^{32}$ S. Plimpton, J. Comput. Phys. 117, 1 (1995).

${ }^{33}$ D. J. Evans and B. L. Holian, J. Chem. Phys. 83, 4069 (1985).

${ }^{34}$ J. Janeček, J. Phys. Chem. B 110, 6264 (2006).

${ }^{35}$ D. Frenkel and B. Smit, Understanding Molecular Simulations (Academic, 2002) pp. 168-172.

${ }^{36}$ B. J. Block, S. K. Das, M. Oettel, P. Virnau, and K. Binder, J. Chem. Phys. 133, 154702 (2010)

${ }^{37}$ J. H. Weijs, A. Marchand, B. Andreotti, D. Lohse, and J. H. Shoeijer, Phys. Fluids 23, 022001 (2011).

${ }^{38}$ G. Scocchi, D. Sergi, C. D’Angelo, and A. Ortona, Phys. Rev. E 84, 061602 (2011).

${ }^{39}$ J. Hautman and M. L. Klein, Phys. Rev. Lett. 67, 1763 (1991).

${ }^{40}$ W. Mar, J. Hautman, and M. L. Klein, Comput. Mater. Sci. 3, 481 (1995).

${ }^{41}$ M. J. de Ruijter, T. D. Blake, and J. De Coninck, Langmuir 15, 7836 (1999).

${ }^{42}$ C. D. Daub, D. Bratko, K. Leung, and A. Luzar, J. Phys. Chem. C 111, 505 (2007).

${ }^{43}$ N. Giovambattista, P. G. Debenedetti, and P. J. Rossky, J. Phys. Chem. B 111, 9581 (2007).

${ }^{44}$ N. Giovambattista, P. G. Debenedetti, and P. J. Rossky, Proc. Natl. Acad. Sci. U.S.A. 106, 15181 (2009).

${ }^{45}$ R. Godawat, S. N. Jamadagni, and S. Garde, Proc. Natl. Acad. Sci. U.S.A. 106, 15119 (2009).

${ }^{46}$ N. Shenogina, R. Godawat, P. Keblinski, and S. Garde, Phys. Rev. Lett. 102, 156101 (2009)

${ }^{47}$ C. D. Daub, J. H. Wang, S. Kudesia, D. Bratko, and A. Luzar, Faraday Discuss. 146, 67 (2010).

${ }^{48}$ J. D. Halverson, C. Maldarelli, A. Couzis, and J. Koplik, Soft Matter 6, 1297 (2010).

${ }^{49}$ T. A. Ho, D. Argyris, D. V. Papavassiliou, A. Striolo, L. L. Lee, and D. R. Cole, Mol. Simul. 37, 172 (2011).

${ }^{50}$ T. A. Ho, D. V. Papavassiliou, L. L. Lee, and A. Striolo, Proc. Natl. Acad. Sci. U.S.A. 108, 16170 (2011)

${ }^{51}$ J. A. Ritchie, J. Seyed Yazdi, D. Bratko, and A. Luzar, J. Phys. Chem. C 116, 8634 (2012).

${ }^{52}$ S. K. Das and K. Binder, EPL 92, 26006 (2010).

${ }^{53}$ D. Bratko, C. D. Daub, K. Leung, and A. Luzar, J. Am. Chem. Soc. 129, 2504 (2007).

${ }^{54}$ Y. Wu, H. L. Tepper, and G. A. Voth, J. Chem. Phys. 124, 024503 (2006).

${ }^{55}$ C. D. Daub, D. Bratko, and A. Luzar, J. Phys. Chem. C 115, 22393 (2011).

${ }^{56}$ G. Taubin, IEEE Trans. Pattern Aanl. Mach. Intell. 13, 1115 (1991).

${ }^{57}$ V. H. Dalvi and P. J. Rossky, Proc. Natl. Acad. Sci. U.S.A. 107, 13603 (2010).

${ }^{58}$ C. D. Bain and G. M. Whitesides, Adv. Mater. 1, 110 (1989).

${ }^{59}$ L. H. Dubois and R. G. Nuzzo, Annu. Rev. Phys. Chem. 43, 437 (1992).

${ }^{60}$ D. M. Huang and D. Chandler, Phys. Rev. E 61, 1501 (2000).

${ }^{61}$ H. S. Ashbaugh and M. E. Paulaitis, J. Am. Chem. Soc. 123, 10721 (2001).

${ }^{62}$ J. Wu, J. Phys. Chem. B 113, 6813 (2009).

${ }^{63}$ J. Rafiee, X. Mi, H. Gullapalli, A. V. Thomas, F. Yavari, Y. Shi, P. M. Ajayan, and N. A. Koratkar, Nature Mater. 11, 217 (2012).

${ }^{64}$ D. Bratko, B. Jönsson, and H. Wennerström, Chem. Phys. Lett. 128, 449 (1986).

${ }^{65}$ Effects of ionic functionalization of graphane are described in D. Vanzo, D. Bratko, and A. Luzar, "Tunable wetting of surfaces with ionic functionalities," J. Phys. Chem. C (in press). 